\title{
HIGHLIGHTS
}

HEMATOLOGY

\section{MYC and chemotherapy response}

Diffuse-large B-cell lymphomas (DLBCL) are a heterogeneous group of tumors with distinct morphological, molecular and clinical subtypes. Approximately $5-10 \%$ of patients with DLBCL harbor $M Y C$ oncogene rearrangements and patients with these genetic alterations who are treated with cyclophosphamide, doxorubicin, vincristine and prednisone (CHOP) chemotherapy have a poor prognosis. However, no studies have assessed the prognostic significance of $M Y C$ rearrangements in patients treated with the more recent regimen of CHOP chemotherapy and rituximab. A recent study has now confirmed that MYC rearrangements in patients with DLBLC treated with rituximab chemotherapy are associated with a poor prognosis. "The present study is the first to evaluate whether the presence of a MYC gene rearrangement is still of clinical relevance in patients treated in the rituximab-CHOP era" comment the researchers.

Savage and coauthors determined the frequency of MYC rearrangements in an unselected group of patients with DLBCL and correlated this with clinical and pathologic features. They also assessed the prognostic impact of these rearrangements in patients treated with rituximab chemotherapy. Fluorescence in situ hybridization (FISH) identified 12 patients of 135 who were positive for the MYC rearrangement. Patients with this genetic rearrangement were more likely to have tumors with a high proliferation rate.

The WHO lymphomas classification recognizes two molecular DLBCL subtypes based on the cell of origin: germinal center B-cell-like (GCB) and activated B-cell-like (ABC), also referred to as non-GCB by immunohistochemistry analysis. There was no difference in the frequency of GCB and non-GCB subtypes according to MYC status. An inferior progression-free survival (PFS) was observed for patients treated with rituximab-CHOP chemotherapy who harbored MYC rearrangement compared with those lacking this genetic alteration (66\% versus $31 \%, P=0.006$ ). Moreover, a worse overall survival was also seen in patients with $M Y C$ rearrangements treated with rituximab-CHOP $(72 \%$ versus $33 \%, P=0.016)$. Multivariate analysis confirmed the importance of $M Y C$ rearrangement as a prognostic factor for a worse PFS (hazard ratio 3.28, $P=0.003$ ) and overall survival (hazard ratio $2.98, P=0.011$ ).

Central nervous system (CNS) relapse was also evaluated and the presence of $M Y C$ rearrangement was shown to be predictive of CNS relapse in patients treated with rituximab-CHOP. This correlation was assessed in multivariate analysis and was shown to remain significant after adjusting for the presence of other risk factors such as multiple extranodal sites or testicular involvement.
4 ...all patients with DLBCL should undergo FISH or karyotype analysis to determine their MYC status... 77

The researchers comment that the diagnosis of DLBCL with MYC rearrangements is likely to be underappreciated. "Given the lack of defining risk factors, FISH analysis for MYC rearrangements should be performed on all patients with DLBCL." Since CNS relapse is a known risk factor in patients with Burkitt lymphoma, chemoprophylaxis is usually included in treatment regimens for this disease. As there was an increased risk of CNS relapse in patients with $M Y C$ rearrangements treated with rituximab$\mathrm{CHOP}$, and the worse prognosis in the patients, this study "raises the question as to whether this population should be treated with Burkitt-lymphoma-type regimens" conclude the researchers. Since MYC rearrangements define a subgroup of patients with an increased risk of CNS relapse and are less likely to be cured with rituximab-CHOP chemotherapy, all patients with DLBCL should undergo FISH or karyotype analysis to determine their MYC status, because regimens used for the treatment of Burkitt lymphoma might be more appropriate.

\section{Lisa Hutchinson}

Original article Savage, K. J. et al. MYC gene
rearrangements are associated with a poor prognosis in
diffuse large B-cell lymphoma patients treated with R-CHOP
chemotherapy. Blood 114, 3533-3537 (2009)

